\title{
An Input Shaping Controller Enabling Cranes to Move Without Sway
}

by

E. Kriikku

Westinghouse Savannah River Company

Savannah River Site

Aiken, South Carolina 29808

N. Singer

Convolve, Inc.

NY USA

W. Singhose

Massachusetts institute of Technology

MA USA

\section{MASTER}

A document prepared for AMERICAN NUCLEAR SOCIETY 7TH TOPICAL MEETING ON ROBOTICS AND REMOTE SYSTEMS at Augusta, GA, USA from 4/27/97 - 5/1/97.

DOE Contract No.

\section{DE-AC09-96SR18500}

DISTRIBUTION OF THIS DOCUMENT IS UNLIMITED

This paper was prepared in connection with work done under the above contract number with the U.S.

Department of Energy. By acceptance of this paper, the publisher and/or recipient acknowledges the U.S. Government's right to retain a nonexclusive, royalty-free license in and to any copyright covering this paper, along with the right to reproduce and to authorize others to reproduce all or part of the copyrighted paper. 


\section{DISCLAMMER}

Portions of this document may be illegible in electronic image products. Images are produced from the best available original document. 


\section{DISCLAIMER}

This report was prepared as an account of work sponsored by an agency of the United States Government. Neither the United States Government nor any agency thereof, nor any of their employees, makes any warranty, express or implied, or assumes any legal liability or responsibility for the accuracy, completeness, or usefulness of any information, apparatus, product, or process disclosed, or represents that its use would not infringe privately owned rights. Reference herein to any specific commercial product, process, or service by trade name, trademark, manufacturer, or otherwise does not necessarily constitute or imply its endorsement, recommendation, or favoring by the United States Government or any agency thereof. The views and opinions of authors expressed herein do not necessarily state or reflect those of the United States Government or any agency thereof.

This report has been reproduced directly from the best available copy.

Available to DOE and DOE contractors from the Office of Scientific and Technical Information, P. O. Box 62, Oak Ridge, TN 37831; prices available from (423) 576-8401.

Available to the public from the National Technical Information Service. U. S. Department of Commerce, 5285 Port Royal Rd., Springfield, VA 22161 


\title{
AN INPUT SHAPING CONTROLLER ENABLING CRANES TO MOVE WITHOUT SWAY
}

\author{
Neil Singer \\ Convolve, Inc. \\ New York, NY \\ William Singhose \\ Dept. of Mechanical Engineering \\ Massachusetts Institute of Technology \\ Cambridge, MA \\ Eric Kriikku \\ Savannah River Technology Center \\ Westinghouse Savannah River Company \\ Aiken, SC
}

\begin{abstract}
A gantry crane at the Savannah River Technology Center was retrofitted with an Input Shaping controller. The controller intercepts the operator's pendant commands and modifies them in real time so that the crane is moved without residual sway in the suspended load. Mechanical components on the crane were modified to make the crane suitable for the anti-sway algorithm. This paper will describe the required mechanical modifications to the crane, as well as, a new form of Input Shaping that was developed for use on the crane. Experimental results are presented which demonstrate the effectiveness of the new process. Several practical considerations will be discussed including a novel (patent pending) approach for making small, accurate moves without residual oscillations.
\end{abstract}

\section{INTRODUCTION}

The use of gantry cranes in nuclear environments requires limitation of both the swing during the motion and the residual oscillation. More commonplace uses of gantry cranes, such as loading ship cargoes, also require limitation of oscillation for throughput and safety. Experienced crane operators attempt to eliminate the residual sway by causing a deceleration oscillation which cancels the oscillation induced during acceleration, or they may brush the payload against obstacles to damp out the oscillation.

If a computer controller is utilized and cable swing is considered in the control design, the time-optimal commands which results in zero residual vibration can be generated. 1,2 Hoisting of the load during the slew increases the difficulty of generating the control because the system frequency is time-varying. Optimal controls based on a nonlinear model are even more difficult to generate. ${ }^{3}$ One method for developing optimal controls divides the motion into five fundamental sections. The optimal control for each section is then derived and pieced together by satisfying boundary conditions. 4 Even when optimal commands can be generated, implementation is usually impractical because the boundary conditions (the move length) must be known at the beginning of the move. When feedback is available, adaptive controllers and combination open- and closedloop control is possible. ${ }^{5,6}$

In this paper the feedforward control technique of Input Shaping is investigated. Input Shaping is easier to derive and implement than time-optimal control schemes and does not require the feedback mechanisms of closedloop and adaptive controllers. Rather than attempt to obtain exactly zero residual vibration (which is impossible on real machines), the version of Input Shaping described here yields non-zero, but low levels of sway.

Input Shaping is implemented in real time by convolving the command signal with an impulse sequence (an input shaper). This process is illustrated in Figure 1 with a step input and an input shaper containing three positive impulses. Note that the shaped input that results from the convolution has a rise time which is longer than the unshaped input by an amount equal to the duration of the input shaper. Similarly, the deceleration time of a shaped command is longer than the unshaped command.

Input shaping is a form of Finite Impulse Response (FIR) filtering that places zeros near the locations of the flexible poles of the original system. The impulse amplitudes are equivalent to the filter coefficients. The impulse amplitudes and time locations are determined by satisfying a set of constraint equations. Input Shaping has been shown to be effective for controlling oscillation of gantry cranes with or without hoisting of the load. 7,8

When input shapers are designed in the z-plane (taking into account the digital sampling rate), the filter 


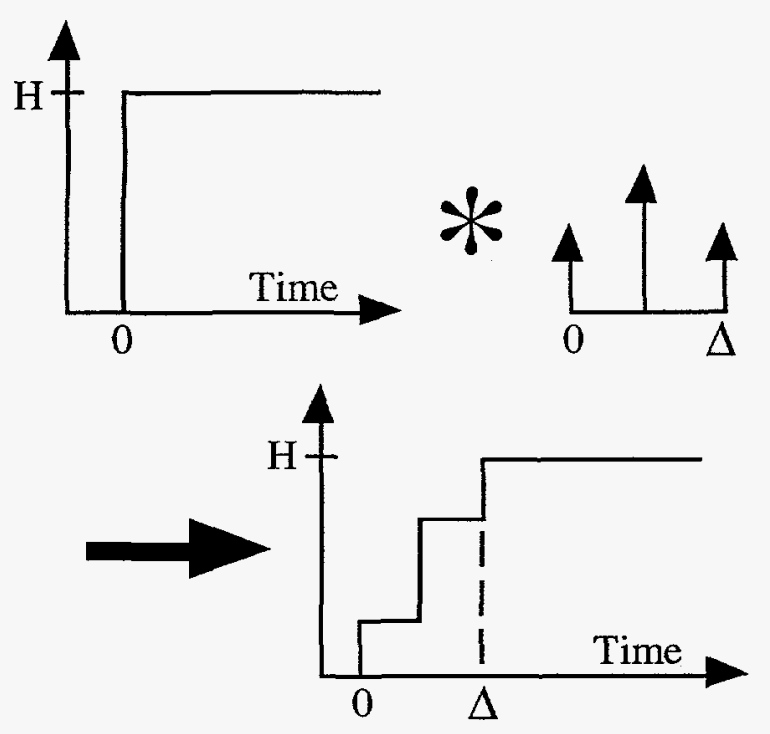

Figure 1: Input Shaping a Step Input.

coefficients can take on large positive and negative values. This occurs when the shaper duration is small compared to the period of vibration being targeted for elimination. When the filters are used to generate a shaped command, the command can greatly exceed the magnitude of the desired command. This will often lead to saturation of the actuators and poor vibration reduction. For example, Figure 2 shows a step input convolved with a shaper containing impulses with amplitudes of $2,-2$, and 1 . The shaped command reaches the same final setpoint, but it initially requests double the actuator effort.

Several schemes have been proposed for dealing with the problem of negative impulses. In one approach, the spacing between the impulses in increased (by multiples of the sampling period) until the resulting shaper is long enough so that it contains only positive impulses. ${ }^{9}, 10$ Another technique allows negative impulses, but limits their magnitude to low enough levels to avoid saturation. 11-13 Yet another scheme, which has shown benefit for cranes, ${ }^{14}$ deals with actuator saturation by adding poles to the shaping filter. The result is an Infinite Impulse Response (IIR) filter that places zeros near the system flexible poles and poles somewhere else in the z-plane so that the transfer function of the filter does not greatly exceed unity.

This paper describes a new procedure for designing input shapers which takes into account the special properties of gantry cranes. The properties utilized are: the deceleration time the operators are accustomed to, the single-mode nature of the dynamics, and the known frequency range of the single, dominant mode. By using these three properties, input shapers can be derived which deliver better performance than previously proposed Input Shaping schemes.

The new input shapers were implemented and tested on a 15 ton gantry crane at the Savannah River

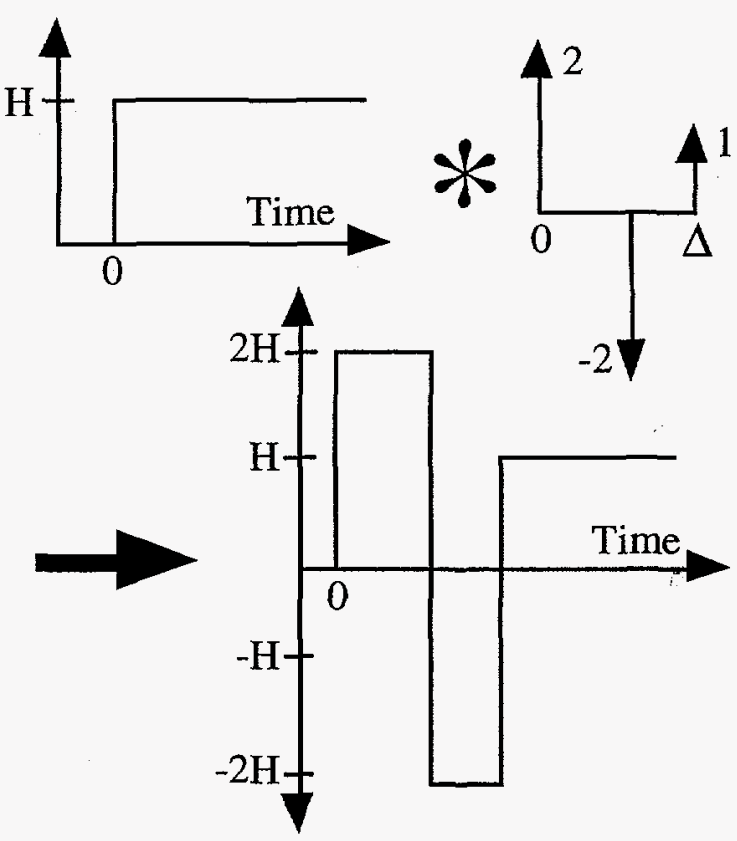

Figure 2: Large Positive and Negative Impulses can Lead to Actuator Saturation.

Technology Center (SRTC). The crane bridge travel is $85 \mathrm{ft}$., the trolley travel is $43 \mathrm{ft}$., and the hook travel is $29 \mathrm{ft}$. In order to implement shaping, some mechanical modifications of the crane were required. The next section describes these modifications. The new shaping scheme is then described and experimental results are shown. The section following the experimental results describes a further modification which allows small step motions to be performed with a swing-free response.

\section{MECHANICAL MODIFICATIONS}

The unmodified crane used two 460 volt $A C$ induction motors on the bridge and one on the trolley. The Input Shaping technique that was implemented requires that variable velocity commands are issued to the crane. The motors on the crane were upgraded to use 460 Volt Baldor AC Vector drives. The drives were programmed to track a velocity signal sent as an analog voltage input (110 Volts DC). All other trajectory features of the drives were turned off. The motors were sized to be roughly equivalent to the pre-existing motors in both horsepower and maximum velocity. Several gearboxes were also changed for physical compatibility with the motors and for general maintenance considerations; they were not required for Input Shaping.

Input Shaping was implemented with a Convolve, Inc. Input Shaping Crane Controller. This controller is a hardware module that is based on an Allen-Bradley SLC-500 modular PLC Rack. The SLC-500 modular system allows selection of input and output modules so that the controls can be easily customized to any crane configuration without hardware redesign. The controller uses these industrially-hardened PLC and I/O modules for 


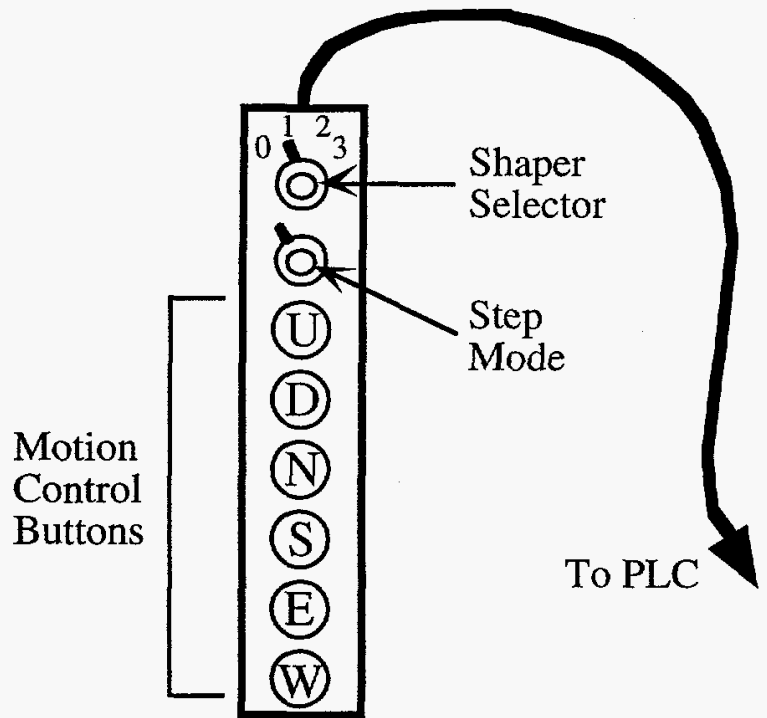

Figure 3: Operator Pendant.

handling all of the crane input and output signals. A proprietary Convolve module is added to the rack to enable Input Shaping. The shaped commands are processed internally and output as analog velocity commands by the SLC-500 analog output module.

The operator presses six on-off buttons on a pendant, shown in Figure 3, to move the crane throughout its workspace (Up/Down, North/South, and East/West). Two additional, four-position switches were added to the crane pendant. The first switch selects the input shaper to be used and the second switch selects Step Mode (described in Section V). The shaping control knob allows the operator to select one of four possible states: no shaping, shaper \#1, shaper \#2, or shaper \#3. Using the approach described in the next section, three fixed input shapers were designed to eliminate vibration throughout the entire workspace of the crane.

The Convolve Input Shaping technology is robust to variations in the sway frequency of the crane. Therefore, the technique was implemented on the crane without the need (and expense) of measuring the cable length and adjusting the input shapers based on the measurement.

\section{FIXED-DURATION INPUT SHAPERS}

Input shapers are designed by constructing and solving a set of constraint equations while minimizing a performance criteria. The constraint equations typically set limits on: residual vibration amplitude, robustness to modeling errors, and actuator effort. The performance criteria which is minimized most often is the rise time, which is equivalent to the duration of the shaper.

The constraint on residual vibration amplitude can be conveniently expressed as the ratio of residual vibration amplitude with shaping to that resulting from a step input. For a mode of natural frequency, $\omega$, and

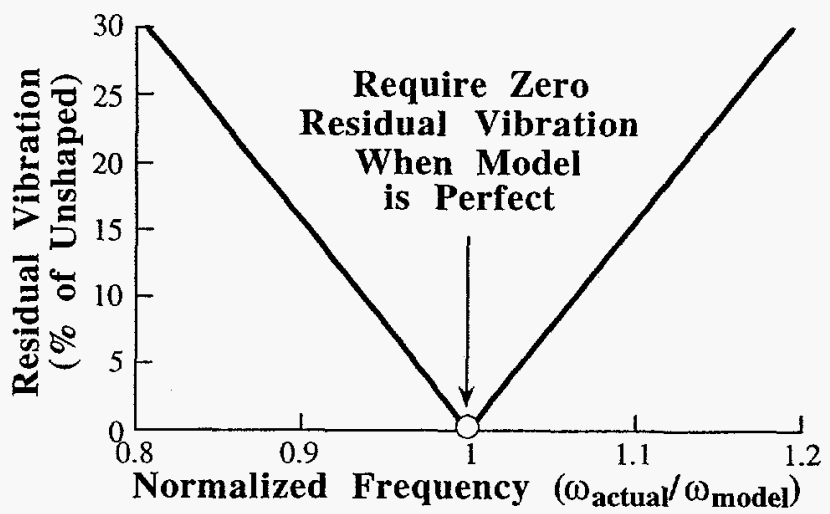

Figure 4: Sensitivity Curve of $\mathrm{ZV}$ Shaper.

damping ratio, $\zeta$, this percentage vibration is given by: ${ }^{15}$

$$
\mathrm{V}(\omega, \zeta)=\mathrm{e}^{-\zeta \omega \mathrm{t}_{\mathrm{n}}} \sqrt{[\mathrm{C}(\omega, \zeta)]^{2}+[\mathrm{S}(\omega, \zeta)]^{2}}
$$

where,

$$
\begin{aligned}
& \mathrm{C}(\omega, \zeta)=\sum_{\mathrm{i}=1}^{\mathrm{n}} \mathrm{A}_{\mathrm{i}} \mathrm{e}^{\zeta \omega \mathrm{t}_{\mathrm{i}}} \cos \left(\omega \sqrt{1-\zeta^{2}} \mathrm{t}_{\mathrm{i}}\right) . \\
& \mathrm{S}(\omega, \zeta)=\sum_{\mathrm{i}=1}^{\mathrm{n}} \mathrm{A}_{\mathrm{i}} \mathrm{e}^{\zeta \omega \mathrm{t}_{i}} \sin \left(\omega \sqrt{1-\zeta^{2}} \mathrm{t}_{\mathrm{i}}\right) .
\end{aligned}
$$

$A_{i}$ and $t_{i}$ are the amplitudes and time locations of the impulses, and $\mathrm{n}$ is the number of impulses in the input shaper. Because (1) is a function of the impulse amplitudes and time locations, it can be used to design an input shaper which will limit the residual vibration to some small value, $V$, at the mode described by $\omega$ and $\zeta$. If $V$ is set equal to zero and (1) is used to design an input shaper, then the resulting shaper is called a Zero Vibration (ZV) shaper.

In practice, $\mathrm{ZV}$ shapers can be very sensitive to modeling errors. To examine this possibility, the amplitude of residual vibration can be plotted as a function of the modeling errors. Figure 4 shows such a sensitivity curve for the $\mathrm{ZV}$ shaper. Notice that the vibration amplitude increases rapidly as the actual frequency deviates from the modeling frequency.

Multiple versions of (1) can be used to suppress a range of frequencies, ${ }^{16}$ thereby obtaining robustness to modeling errors. Each version of (1) is unique in that different values for the modal parameters are used. For example, suppose a gantry crane like the one sketched in Figure 5, is known to operate with a cable length, $L$, ranging from $20 \mathrm{ft}$. to $4 \mathrm{ft}$. Using the linearized model of this system, the frequency varies between $0.2 \mathrm{~Hz}$ and $0.45 \mathrm{~Hz}(\omega=\sqrt{\mathrm{g} / \mathrm{L}})$. Furthermore, suppose that it is required to reduce the residual oscillation to $5 \%$ of the level obtained during normal operation. To design a shaper which meets these requirements, we can set $\mathrm{V}=$ 0.05 and use $\mathrm{N}$ versions of (1) applied at various frequencies throughout the range from $0.2 \mathrm{~Hz}$ to 0.45 $\mathrm{Hz}$. A numerical optimization is then performed to obtain a shaper which satisfies the constraints while 


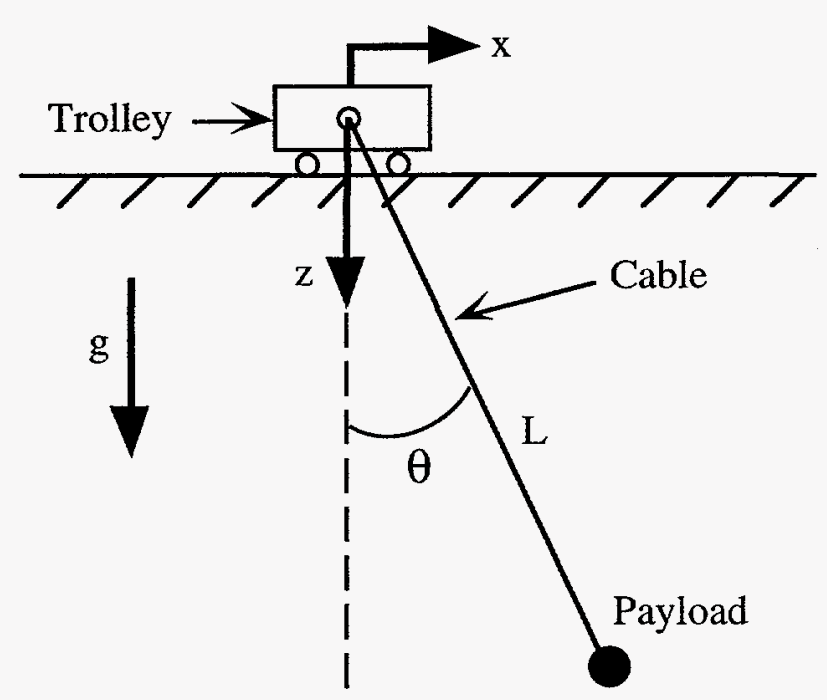

Figure 5: Model of a Planar Gantry Crane.

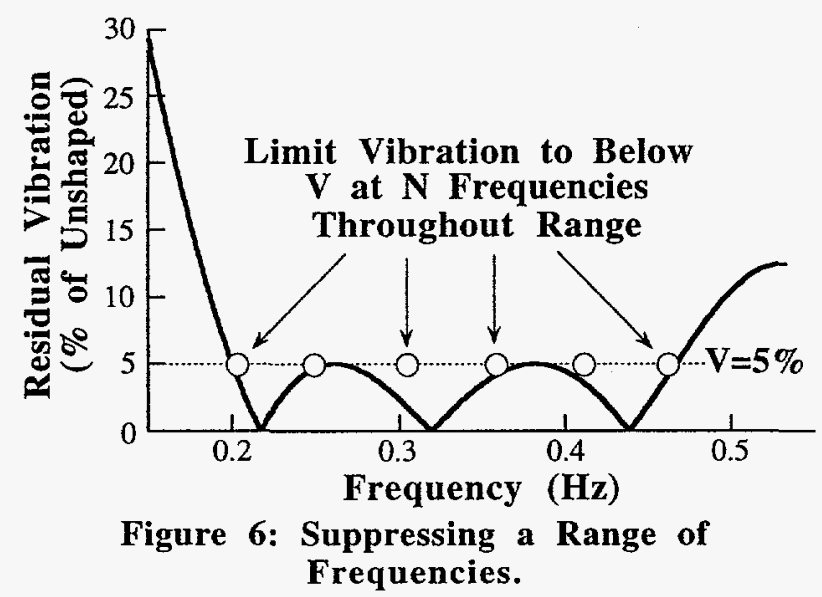

being of minimum length. This process, demonstrated in Figure 6, is called specified insensitivity (SI) design. 16

Note that this process does not guarantee the residual amplitude will never exceed $\mathrm{V}$ throughout the frequency range; it only guarantees it will be below $\mathrm{V}$ at the frequencies where (1) is applied. However, if the sampling frequencies are made close enough together, the residual amplitude is effectively limited over the entire range. In practice, $\mathrm{N}$ does not have to be large, a value between 3 and 10 is often sufficient.

In order for the shaped commands to perform the same rigid-body motion as the unshaped commands, the impulse amplitudes must sum to one. In equation form:

$$
\sum_{i=1}^{n} A_{n}=1
$$

This requirement is easily justified by examining the case when the unshaped command is a step input of amplitude $H$. When a step input is convolved with a sequence of positive impulses, the resulting command is a staircase, as shown in Figure 1. The final value of the staircase is equal to $\mathrm{H}$ times the summation of impulses

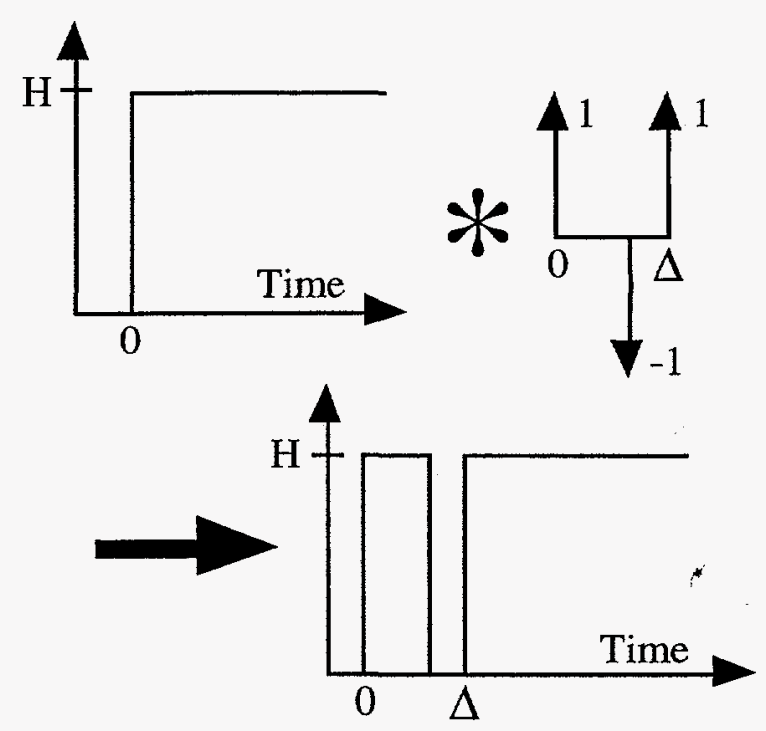

Figure 7: Shaping with a UM Shaper.

amplitudes. Therefore, to reach the same final setpoint, the impulse amplitudes must sum to one.

The constraints given by (1) and (2) can be used to limit the flexible-body dynamics and to set the rigid-body motion. However, if (1) and (2) are satisfied while minimizing the shaper length $\left(t_{n}\right)$, the optimization fails to find a practical solution because the impulse amplitudes are driven towards positive and negative infinity. In effect, the actuator limits of the real system have not been taken into account. A simple way to avoid this problem is to require all impulses to have a positive value. ${ }^{15}$ Given this constraint, the shaped input will never exceed the magnitude of the unshaped input.

However, if the amplitudes are allowed to take on negative values, the rise time can be decreased. ${ }^{11-13}, 17$ In particular, it has been shown that limiting the impulse values to \pm 1 yields input shapers which give a fast response while still satisfying actuator limits in most cases. Figure 7 shows a step input shaped with a Unity Magnitude (UM) shaper containing three impulses. The shaper duration, $\Delta$, is less for a UM shaper than for a shaper containing all positive impulses. Note that the shaper produces a command which is either full on or off. The UM shaper moves a system faster than a shaper with all positive pulses because it utilizes more of the available actuator effort.

A drawback to UM shapers is that they have a tendency to excite unmodeled high modes. 11,12 Fortunately, gantry cranes are usually single-mode systems with no significant higher modes. Therefore, the technique described in this paper will use the UM amplitude constraint given by:

$$
A_{i}=(-1)^{i+1} \quad i=1, \ldots, n .
$$

where $\mathrm{n}$ is odd. Note that a shaper satisfying (3) automatically satisfies (2). 
In previous work, input shapers have been designed by satisfying various combinations of residual vibration, robustness, and impulse amplitude constraints while minimizing the shaper length. The shapers are described by the constraint equations used to design them. For example, a shaper designed using the unity-magnitude (UM) constraint and the zero vibration (ZV) constraint would be called a UM ZV shaper.

The duration of a shaper designed by minimizing the shaper length is a function of the vibration period. When the shapers are used to eliminate vibration in remote systems, such as gantry cranes, the shaped command profile lags the unshaped profile by the duration of the shaper. (See again Figure 1.) In effect, when the operator requests the system to stop, the system decelerates for a period equal to the shaper duration. Deceleration periods are common on cranes without Input Shaping. The motors are ramped down so as to decrease residual sway. Without shaping, these deceleration periods are constant and the operator becomes accustomed to the lag. Using previously proposed input shaping schemes, the deceleration period would vary with the system frequency.

We now propose a new input shaper design algorithm which results in a fixed-duration (FD) shaper, regardless of the system oscillation period. In this approach, the constraint equations are multiple versions of (1), (2) , (3), and a fixed shaper duration:

$$
t_{n}=\Delta_{t} \text {. }
$$

With the shaper duration fixed at $\Delta_{t}$, it no longer makes sense to minimize the shaper length, so instead, the robustness of the shaper is maximized. That is, the range of frequencies over which the residual vibration can be kept below $\mathrm{V}$ is maximized.

Given that the suppressed frequency range is being maximized, there is no guarantee that the vibration can be suppressed for all cable lengths with a single input shaper. To counter this difficulty, several shapers can be used, each one is designed to suppress vibration in a subset of the operating range.

To demonstrate the FD shaper design process, suppose that we are designing shapers for the crane that varies its cable length from $20 \mathrm{ft}$. down to $4 \mathrm{ft}$. The constraint equations consist of (2)-(4) and (1) applied at the frequency, $\omega_{\mathrm{l}}$, corresponding to $20 \mathrm{ft}$. An additional version of (1) is applied at an unknown frequency, $\omega_{h}$, that is higher in value than $\omega_{1}$. The frequencies between $\omega_{h}$ and $\omega_{1}$ are suppressed by $N$ additional versions of (1). An optimization is then performed to obtain the shaper that maximizes $\omega_{\mathrm{h}}$.

Suppose that $\omega_{\mathrm{h}}$ corresponds to a cable length of 8 ft. That is, a single shaper cannot suppress vibration over the entire range of cable lengths. In this case, the optimization is performed again, but this time $\omega_{\mathrm{h}}$ plays the role of $\omega_{1}$. If the new value, $\omega_{\mathrm{h} 2}$, resulting from the second optimization is above the frequency corresponding to $4 \mathrm{ft}$., then the shaper design process is complete. The

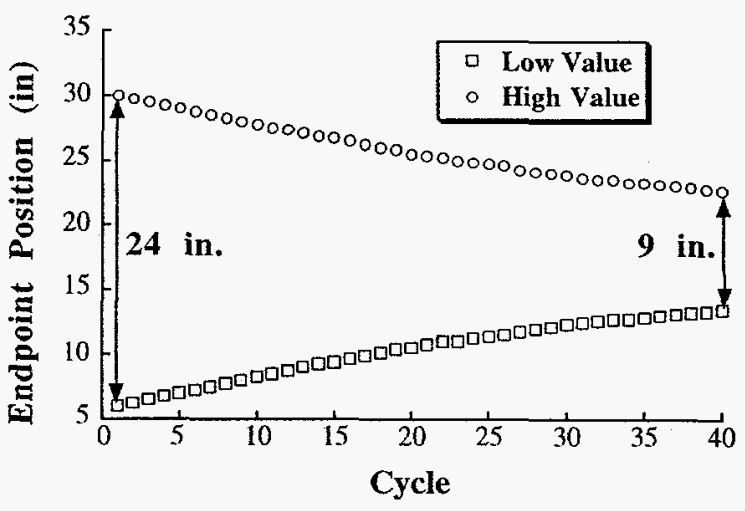

Figure 8: Measured Peak-to-Peak Oscillation of Crane with a Cable Length of $28 \mathrm{ft}$.

shaper resulting from the first optimization is used to move the crane when the cable is between $20 \mathrm{ft}$. and 8 ft., while the second shaper is used when the cable is less than $8 \mathrm{ft}$. in length.

The fixed-duration shaper design algorithm can be summarized as follows:

1) Pick a shaper length (deceleration period), $\Delta_{t}$.

2) Select a desired limit on the percentage residual vibration amplitude, $V$.

3) Require the vibration to be below $V$ at the lowest possible frequency, $\omega_{\mathbf{l}}$, (the longest cable length).

4) Perform an optimization which maximizes the frequency range over which the residual amplitude can be kept below V. The output of the optimization is an input shaper and the maximum suppressed frequency, $\omega_{\mathrm{h}}$.

5) If the frequency range covers the entire workspace, then terminate the algorithm. Otherwise, start at step 3 and replace $\omega_{1}$ with $\omega_{\mathrm{h}}$.

The product of the algorithm is one or more fixedduration shapers which can be used to suppress vibration throughout the workspace.

\section{EXPERIMENTAL RESULTS}

The above procedure for designing fixed-duration shapers was performed for a gantry crane at SRTC. To measure the residual oscillation of the crane, a yard stick was attached to the crane hook and the response was recorded on videotape. By placing a straightedge on the surface of the video monitor, the peak-to-peak residual oscillation was measured. For long cable lengths (endpoint near ground), the setup gave a resolution of 1/8 in. As the cable was shortened and the endpoint moved away from the camera, the resolution deteriorated. At short cable lengths $(<15 \mathrm{ft})$, the resolution was approximately $1 / 4-3 / 8$ in.

The damping ratio of the SRTC crane was determined by moving the crane and recording the peakto-peak oscillation amplitude over 40 cycles of vibration. Figure 8 shows the values of the experimental measurements with a cable length of $28 \mathrm{ft}$. Even after 


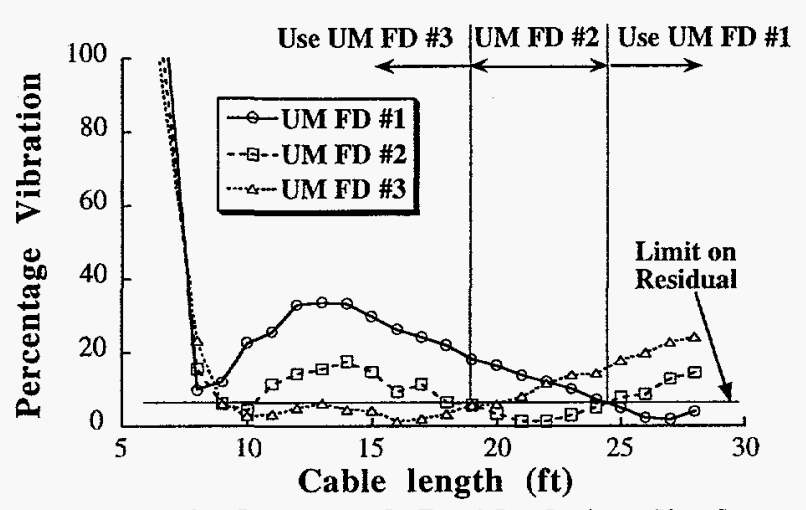

Figure 9: Measured Residual Amplitude Using UM FD Shapers.

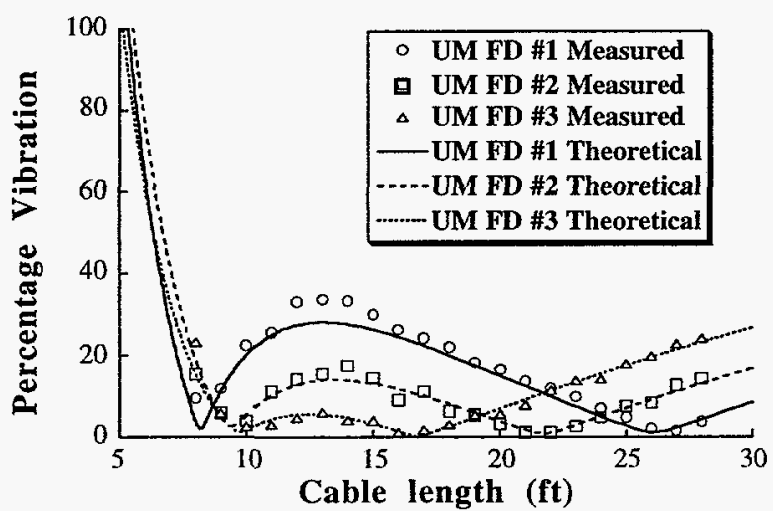

Figure 10: Comparison of Theoretical and Measured UM FD Residual Amplitude.

40 cycles (nearly 4 minutes), the peak-to-peak oscillation is still 9 in. An exponential curve fit to the data revealed that the damping ratio was approximately 0.004 . If the cable length is very short ( $5 \mathrm{ft}$. or less), then the damping ratio goes up considerably, to approximately 0.1 . Because the damping was so small throughout almost the entire workspace, the damping ratio was modeled as exactly zero.

Using the approximate deceleration period under the standard control system, $\Delta_{t}$ was set equal to 3.0 seconds and the above procedure was used to design three UM FD shapers to span the range of cable lengths from $29 \mathrm{ft}$. to $9 \mathrm{ft}$. (The crane rarely operates with cable lengths less than $9 \mathrm{ft}$. and cannot operate at less than $5 \mathrm{ft}$.) Figure 9 shows the measured percentage residual for each of the UM FD shapers. The percentage residual is obtained by dividing the shaped residual amplitude by the unshaped residual amplitude for the same motion. If the operator switches between the three shapers at $24 \mathrm{ft}$. and $19 \mathrm{ft}$., then the residual vibration can be kept to below 5\% of the unshaped level (for cable lengths down to $9 \mathrm{ft}$.).

Figure 10 compares the measured residual for the UM FD shapers to the theoretical residual amplitudes. Given the uncertainty in the measurement system, there is very good agreement between theoretical and measured values. Notice that the largest deviation between theory

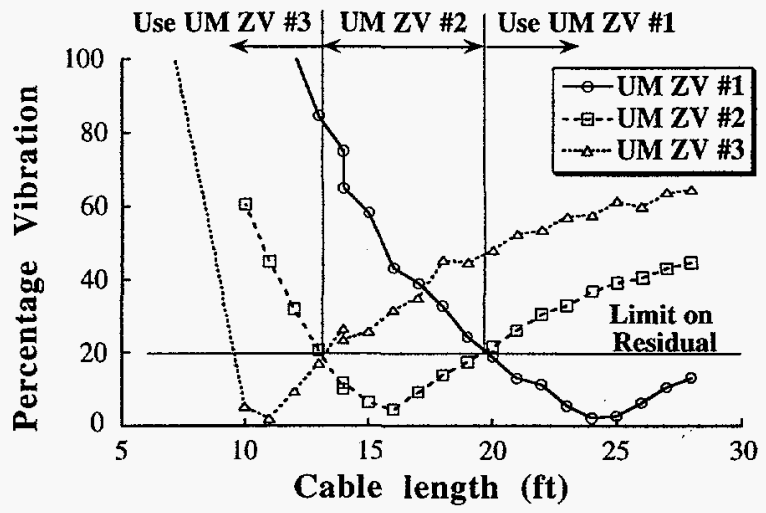

Figure 11: Measured Residual Amplitude Using UM ZV Shapers.

and measured values occurs at short cable lengths. This makes sense, as the measurement system has progressively worse resolution as cable length is decreased and the actual damping ratio deviates more from the modeling value of zero.

To compare the new shaping process with previously proposed methods, three UM ZV shapers were designed to cover the workspace. The shapers were designed to have zero residual vibration at cable lengths of approximately $24 \mathrm{ft}, 16 \mathrm{ft}$, and $11 \mathrm{ft}$. Figure 11 shows the measured percentage residual vibration as a function of cable length. If the operator switches between the three shapers at $20 \mathrm{ft}$ and $13 \mathrm{ft}$, then the residual vibration can be kept to below $20 \%$ of the unshaped level (for cable lengths down to $9 \mathrm{ft}$.).

Comparing Figures 9 and 11, we see that the UM FD shapers provide better sway reduction than the UM ZV shapers throughout the workspace. Unlike the fixedduration shapers, the deceleration lag is different with each UM ZV shaper. The operator must become accustomed to the different deceleration periods. An advantage of the UM ZV shapers is that the deceleration period is shorter; the maximum shaper length is 1.8 seconds.

\section{SMALL STEP MOTION}

By using Input Shaping to make swayless moves, a new mode of operation called Step Mode (Patent Pending) was introduced. Step Mode issues a fixedlength shaped command to the motors so that the crane's load is indexed to a new location without sway. Three different step sizes were programmed into the crane controller. When a crane load is positioned near a final location, the operator is able to switch into Step Mode and bump the load into place without sway. The steps are buffered, therefore, three quick presses of a motion button would move three step distances in one move without stopping.

The input shapers used for step mode are the same as those used for regular crane operation. The Step Mode switch, shown in Figure 3, provides step motion of three different preprogrammed lengths. Step Mode can be used 
without shaping, however, its usefulness is greatly reduced because the crane sways with an amplitude that is nearly the full step size. Step Mode was designed to enable safe and accurate fine positioning of critical loads.

\section{CONCLUSIONS}

A new procedure for designing input shapers for gantry cranes was presented. The procedure takes into account the unique properties of gantry cranes, such as, the single-mode dynamics, the known frequency range, and the standard deceleration period. The new shapers are of fixed duration, so that they give a constant deceleration period. Given the fixed shaper duration, the robustness of the shapers is maximized. The new shaping method was implemented on a gantry crane at the Savannah River Technology Center. Experimental results show that the method greatly reduces residual oscillations and closely matches the theoretically predicted performance.

\section{REFERENCES}

1. J. W. Auernig and H. Troger, "Time Optimal Control of Overhead Cranes with Hoisting of the Load," Automatica, (July), pp. 437-446, 1987.

2. A. R. Golafshani and J. D. Aplevich, "Computation of Time-Optimal Trajectories for Tower Cranes," IEEE Conference on Control Applications, , pp. 1134-1139, 1995.

3. K. A. F. Moustafa and A. M. Ebeid, "Nonlinear Modeling and Control of Overhead Crane Load Sway," Transactions of the ASME, Vol. 110 (September), pp. 266-271, 1988.

4. Y. Sakawa and Y. Shindo, "Optimal Control of Container Cranes," Automatica, Vol. 18 (3), pp. 25766, 1982.

5. H. Butler, G. Honderd and J. V. Amerongen, "Model Reference Adaptive Control of a Gantry Crane Scale Model," IEEE Control Systems, (January), pp. 5762, 1991.

6. K. Sato and Y. Sakawa, "Modeling and Control of a Flexible Rotary Crane," Int. Journal of Control, Vol. 48 (5), pp. 2085-2105, 1988.

7. M. W. Noakes and J. F. Jansen, "Generalized Inputs for Damped-Vibration Control of Suspended Payloads," Robotics and Autonomous Systems, Vol. 10 (2), pp. 199-205, 1992.

8. W. E. Singhose, L. J. Porter and W. P. Seering, "Input Shaped Control of a Planar Gantry Crane with Hoisting," Submitted to the 1997 American Control Conf.

9. S. D. Jones and A. G. Ulsoy, "Control Input Shaping for Coordinate Measuring Machines," Proc. of the American Control Conference, Baltimore, MD, Vol. 3, pp. 2899-2903, 1994.

10. T. D. Tuttle and W. P. Seering, "A Zero-placement Technique for Designing Shaped Inputs to Suppress
Multiple-mode Vibration," Proc. of the American Control Conference, Baltimore, MD, Vol. 3, pp. 2533-2537, 1994.

11. L. Pao and W. Singhose, "Unity Magnitude Input Shapers and Their Relation to Time-Optimal Control," IFAC World Congress, San Francisco, CA, 1996.

12. W. Singhose, N. Singer and W. Seering, "TimeOptimal Negative Input Shapers," J. of Dynamic Systems, Measurement, and Control, In Press.

13. W. Singhose, N. Singer and W. Seering, "Design and Implementation of Time-Optimal Negative Input Shapers," International Mechanical Engineering Congress and Exposition, DSC 55-1, Chicago, IL, pp. 151-7, 1994.

14. J. T. Feddema, "Digital Filter Control of Remotely Operated Flexible Robotic Structures," American Control Conference, San Francisco, CA, Vol. 3, pp. 2710-2715, 1993.

15. N. C. Singer and W. P. Seering, "Preshaping Command Inputs to Reduce System Vibration," J. of Dynamic Systems, Measurement, and Control, Vol. 112 (March), pp. 76-82, 1990.

16. W. E. Singhose, W. P. Seering and N. C. Singer, "Input Shaping for Vibration Reduction with Specified Insensitivity to Modeling Errors," JapanUSA Symposium on Flexible Automation, Boston, MA, 1996.

17. N. C. Singer, "Residual Vibration Reduction in Computer Controlled Machines," MIT Artificial Intelligence Laboratory Technical Report Number AITR-1030, MIT Artificial Intelligence Lab, 1989. 